\title{
Mid-term Clinical Results of Microendoscopic Decompression for Lumbar Foraminal Stenosis
}

\author{
Mitsunori Yoshimoto, Noriyuki Iesato, Yoshinori Terashima, Katsumasa Tanimoto, Tsutomu Oshigiri, Makoto Emori, \\ Atsushi Teramoto and Toshihiko Yamashita
}

Department of Orthopaedic Surgery, Sapporo Medical University School of Medicine, Sapporo, Japan

\begin{abstract}
:
Introduction: There have been several reports on surgical techniques involving microendoscopy or percutaneous endoscopy for treating lumbar foraminal stenosis (LFS). However, no studies have assessed the mid-term clinical results of endoscopic techniques in spite of their relatively long history. In this study, we report 20 consecutive cases of LFS treated by our microendoscopic technique focusing on clinical results with a follow-up of at least two years.

Methods: Twenty consecutive cases of LFS treated with microendoscopic decompression were followed up at 1, 2, 6, and 12 months postoperatively and annually thereafter. The patients were 14 males and 6 females, and the mean age at the time of surgery was 64.7 years. The Japanese Orthopaedic Association (JOA) score was used as the clinical outcome index.

Results: Of the 20 patients, 16 were monitored successfully for more than 2 years. The follow-up rate was $80.0 \%$, and the mean follow-up period was 66.3 months. The JOA score improved from 13.8 points before surgery to 24.6 points at final follow-up. Revision fusion surgeries were performed in two cases for LFS recurrence.
\end{abstract}

Conclusions: The microendoscopic technique effectively treats LFS.

Keywords:

lumbar foraminal stenosis, minimally invasive surgery, microendoscopic surgery

Spine Surg Relat Res 2019; 3(3): 229-235

dx.doi.org/10.22603/ssrr.2018-0076

\section{Introduction}

There is no standardized diagnostic imaging technique for lumbar foraminal stenosis (LFS), so this disease can be easily overlooked and result in failed back surgery syndrome. In many cases, the whole length of the nerve root, from the spinal canal to the extraforaminal zone, must be decompressed surgically since it is difficult to identify the nerve compression site before surgery; as a result, preserving the posterior elements is difficult. Therefore, total facetectomy combined with spinal fusion using spinal instrumentation is normally performed.

Microendoscopic discectomy (MED), developed by Foley and Smith ${ }^{1}$, is used to treat lumbar disc herniation. Because of recent advances in surgical techniques and instruments, spinal microendoscopy is also used for various conditions such as lumbar spinal stenosis (LSS) and cervical myelopathy ${ }^{2,3}$. For lumbar lateral lesions, especially far-lateral lumbar disc herniation and far-out syndrome, spinal microendo- scopy, which can reach deeper into the body less invasively, is becoming the standard procedure ${ }^{4,5}$.

We previously reported a microendoscopic technique to decompress the entire length of the nerve root, from the spinal canal to extraforaminal zone, while preserving the posterior elements in LFS patients ${ }^{6}$. Similar techniques with microendoscopy or percutaneous endoscopy for treating LFS have been reported by several authors ${ }^{7-9)}$. However, during our literature search using PubMed, we found no studies that have assessed the mid-term clinical results of such endoscopic techniques, in spite of their relatively long history. The mid-term results might suggest problems, such as deterioration of clinical results, because of intervertebral foramen restenosis. In this study, we report 20 consecutive cases of LFS treated by our method focusing on clinical results with a follow-up of at least two years. 

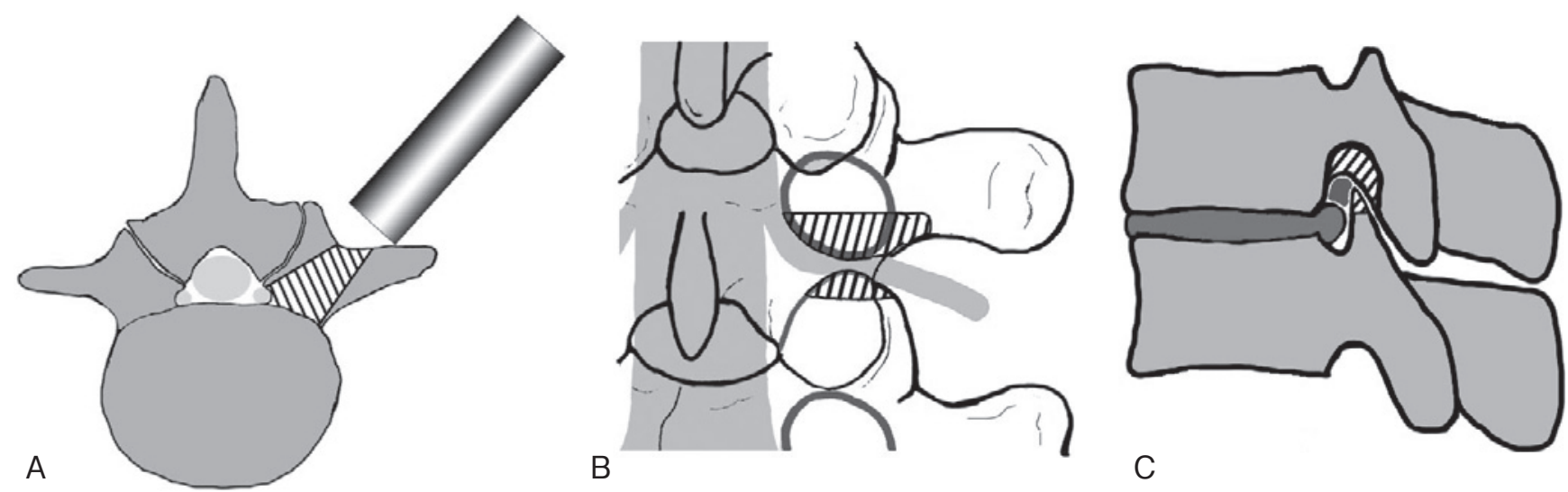

Figure 1. Schematic drawings of the surgical procedure. The shaded portion indicates the bone resection range. The figures are reproduced from [6] (with permission).

A. Axial view. The tubular retractor is placed in an inward-tilting position. The decompression range can reach the lateral border of the spinal canal without damaging posterior elements, such as the facet joints and pars interarticularis.

B. Posterior view. The entire length of the nerve (nerve root, dorsal root ganglion, and spinal nerve), from the lateral border of the spinal canal to the outside of the intervertebral foramen, can be decompressed.

C. Lateral view. The caudal part of the pedicle and cranial part of the superior articular process are removed. Both up-down stenosis and front-back stenosis can be eliminated.

\section{Materials and Methods}

\section{Patient data}

Microendoscopic decompression for LFS was introduced in Sapporo Medical University Hospital in 2008. The first 20 consecutive patients treated with this technique between 2008 and 2016 were followed up at 1, 2, 6, and 12 months postoperatively and annually thereafter. The patients were 14 males and 6 females and the mean age at the time surgery was 64.7 years (range 52-80 years). Seven patients who had degenerative lumbar scoliosis (DLS) with Cobb angles of more than 10 degrees were included in this series. Three patients had undergone posterior decompression for central stenosis previously, but their leg symptoms persisted.

All patients had intermittent claudication with unilateral radicular pain. The preoperative diagnoses were based on detailed neurological examinations, selective nerve root blocks, lumbosacral roentgenographies, and magnetic resonance imaging (MRI) or computed tomography (CT) studies after radicurography ${ }^{6}$. Cases of far-lateral lumbar disc herniation and far-out syndrome were excluded. Patients were candidates for surgery if conservative therapies such as administration of prostaglandin E1 and analgesics, epidural block, and selective nerve root block, for at least three months were ineffective. The most frequently decompressed level was L5-S1 (16 patients), followed by L5-L6 (2 patients) and L4-L5 (2 patients).

\section{Surgical procedure}

All procedures were performed according to the method described by Yoshimoto et $\mathrm{al}^{6}$. Surgery was conducted under general anesthesia with the patient in a prone position on the Hall frame. A longitudinal skin incision of $16 \mathrm{~mm}$ was made 6 to $8 \mathrm{~cm}$ lateral to the midline. The METRx MED system (Medtronic Sofamor Danek, Memphis, TN) was used for surgery. After dissection of the fascia, a dilator with a diameter of $5.3 \mathrm{~mm}$ was inserted toward to the base of the transverse process of the upper vertebra, with the dilator tilted inward at a 45-degree angle or greater, using x-ray fluoroscopy. A guide wire was not generally used. Dilators with larger diameters were inserted sequentially, and a tubular retractor with a diameter of $16 \mathrm{~mm}$ was placed.

The first step was to fully expose the caudal half of the base of the transverse process of the upper vertebra and the lateral edge of the lamina using an electrocautery knife. Since this surgery involves a pinpoint approach, making visualization of the entire area difficult, complete exposure of this region as a landmark is essential to avoid disorientation of the surgeon. Using an air drill, approximately onethird of the pedicle was excavated caudally from the base of the transverse process toward the spinal canal from the inner side, preserving the medial and caudal cortex. The medial and caudal cortex of the pedicle was thinned from the inner side, little by little, using an air drill. The nerve root became visible after the pedicle cortex was resected. Compression factors around the nerve were excised from the spinal canal to the extraforaminal zone, decompressing the nerve root, dorsal root ganglion, and spinal nerve. Up-down stenosis was eliminated by removing the caudal aspect of the pedicle, and front-back stenosis was eliminated by removing the ligamentum flavum, or cranial part of the superior articular process of the lower vertebra. The decompression range can reach the lateral border of the spinal canal without damaging posterior elements, such as the facet joints and pars interarticularis, since the tubular retractor is placed in inwardtilting fashion (Fig. 1). A standard microendoscopic spinal canal decompression was combined for cases with stenosis 
Table 1. Degenerative Lumbar Scoliosis with Cobb Angle $>10$ Degrees and Cobb Angle Changes.

\begin{tabular}{ccc}
\hline Patient number & Pre-operative DLS and Cobb angle & Post-operative DLS and Cobb angle \\
\hline 1 & 12.5 & 19.0 \\
2 & - & - \\
3 & 15.9 & 22.5 \\
4 & 12.0 & 11.8 \\
5 & - & - \\
6 & - & - \\
7 & - & - \\
8 & - & - \\
9 & 16.3 & 17.0 \\
10 & - & - \\
11 & - & - \\
12 & - & - \\
13 & - & - \\
14 & 25.5 & 25.3 \\
15 & - & - \\
16 & - & - \\
\hline
\end{tabular}

DLS=degenerative lumbar scoliosis

of the central canal and lateral recess. Patients were encouraged to walk on the first postsurgical day.

For patients where L5-S1 is affected, the iliac crest may become an obstacle, limiting lateral approaches to the lesion. In such cases, the tubular retractor is inserted from the cranial side, avoiding the iliac crest. CT or MRI should be used to make pre-surgical decisions regarding the direction of insertion of the tubular retractor and the point of skin incision.

\section{Clinical evaluations}

The Japanese Orthopaedic Association (JOA) score (-6-29 points) (Appendix 1) was used as a clinical outcome index. The rate of improvement in JOA score was calculated as follows: (postoperative score-preoperative score) / (29preoperative score) $\times 100 \%$. Cases where JOA score improvement was less than $50 \%$ were recorded as "poor outcome" cases. Required revision surgeries were also recorded. Postoperative DLS progression was evaluated using anteroposterior view radiographs at final follow-up. All clinical and radiographic data were collected prospectively, and this study is a retrospective review of that data.

\section{Statistical analysis}

Changes in JOA scores were analyzed by paired Student's t-test. A $p$-value of less than 0.05 was considered statistically significant.

\section{Ethics}

This study was conducted in compliance with the Declaration of Helsinki. Written informed consent was obtained from all patients before treatment, and the study protocol was approved by the hospital Institutional Review Boards.

\section{Results}

Seven patients underwent the combined procedure because slight stenosis of the lateral recess was seen on CTmyelography. The other 13 patients underwent microendoscopic foraminal decompression alone.

\section{Follow-up status}

Of the 20 patients who underwent the procedure, 4 patients stopped coming to their scheduled clinic visits and did not respond to our letters. The remaining 16 patients, who were successfully monitored prospectively for more than two years, were enrolled in further clinical studies. The follow-up rate was thus $80.0 \%$, and the mean postoperative follow-up period was 66.3 months (range, 24-105 months). Of the 16 patients, 5 had DLS with Cobb angles of more than 10 degrees (Table 1).

\section{Clinical outcomes}

JOA scores improved significantly after surgery in all cases. The mean JOA score was 13.8 points (range, 7-22) before surgery and 24.6 points (range, 14-29) at final follow-up (Fig. 2). The mean improvement rate at the final follow-up was $63.9 \%$ (range, -42.9-100). The mean improvement rates by procedure were $60.1 \%$ in patients who underwent L5-S1 surgery and $67.4 \%$ in patients with different surgeries (i.e., L4-5 or L5-L6). This difference was not significant. Fig. 3 indicates JOA score changes in each case. The significant improvements in JOA scores were maintained until the final follow-up in nine patients. However, JOA scores deteriorated in two patients at final follow-up (patients 1 and 2). Patient 1, who underwent L5-S1 surgery suffered from low back pain caused by progressive DLS, with a Cobb angle change from 12.5 to 19.0 degrees, followed by coronal trunk shift. Patient 2, who underwent L5- 


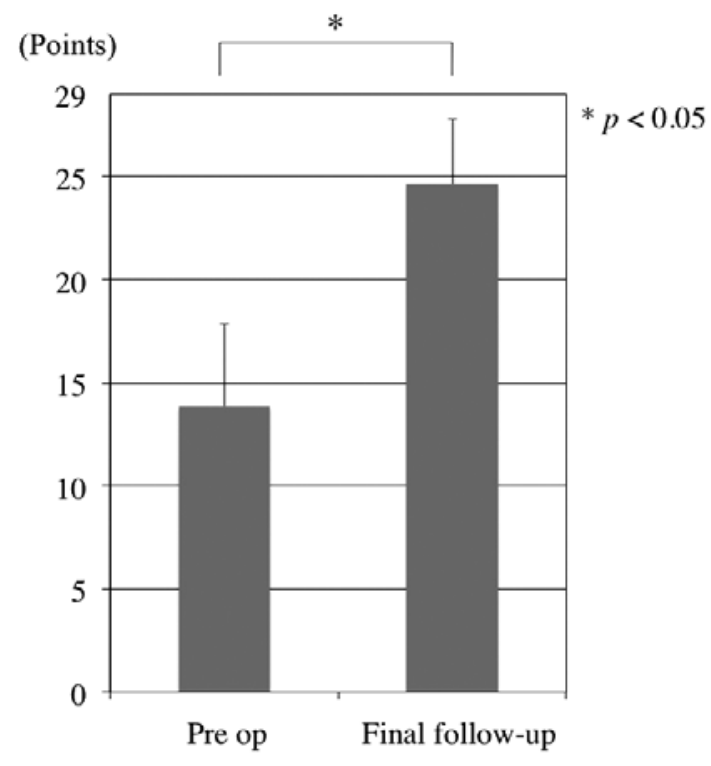

Figure 2. Preoperative and postoperative Japanese Orthopaedic Association scores.

S1 surgery, experienced deterioration in daily living activities because of dislocation after total hip arthroplasty and arteriosclerosis obliterans. Five patients (3-7) experienced deteriorated JOA scores and required revision surgery. DLS progressed in two patients, but no de-novo DLS occurred during follow-up (Table 1).

\section{Revision surgeries (patients 3 to 7)}

Revision surgeries were performed in two cases for LFS recurrence at the operated level (patients 3 and 4), and in three cases for lumbar disc herniation or LSS at other levels (patients 5, 6, and 7). The two patients with LFS recurrence underwent transforaminal lumbar interbody fusion (TLIF) 30 and 34 months after surgery, respectively, and the patients with lumbar disc herniation or LSS at other levels underwent microendoscopic discectomy or decompression 19, 30, and 55 months after surgery.

\section{Cases presentations}

\section{Patient three}

A 64-year-old woman underwent microendoscopic decompression for LFS on the right side at L4-5. Immediately after surgery, the pain in her right leg was greatly improved, and the JOA score improved from 13 points before surgery to 22 points six months after surgery. However, leg pain recurred, the JOA score deteriorated to 9 points, and she underwent TLIF 34 months after decompression surgery. A coronal image of reconstructed three-dimensional CT showed recurrence of foraminal stenosis at the operated level because of DLS progression; the Cobb angle increased from 15.9 to 22.5 degrees (Fig. 4).

\section{Patient four}

A 64-year-old man underwent microendoscopic decom-
(Points)

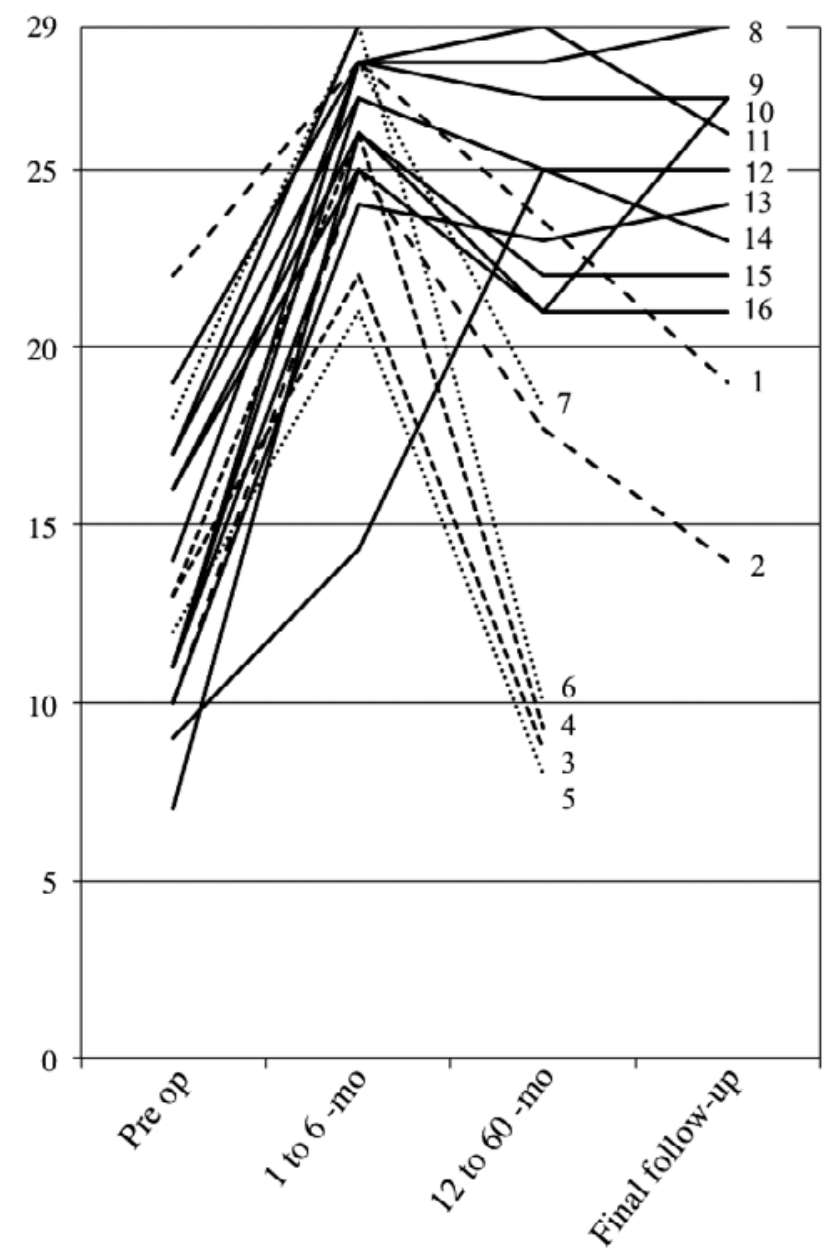

Figure 3. Changes in the Japanese Orthopaedic Association scores in each case. Numbers indicate each patient.

pression for LFS on the right side at L5-S. Immediately after the operation, the pain in his right leg was greatly improved and the JOA score improved from 13 points before surgery to 26 points 6 months after surgery. However, leg pain recurred, the JOA score deteriorated to 9 points, and he underwent TLIF 30 months after decompression surgery. Anteroposterior radiographs did not show DLS progression with 12 degree of Cobb angle (L2-L4), but a coronal image of reconstructed three-dimensional CT showed recurrence of foraminal stenosis at the operated level because of bone regrowth of the L5 pedicle (Fig. 5).

\section{Discussion}

LFS is defined as compression of a nerve at a site between the medial and lateral borders of the pedicle ${ }^{10)}$. Various degenerative changes, such as narrowing of the intervertebral disc space, degenerative lumbar scoliosis, bulging of the intervertebral disc, vertebral body osteophyte formation, anterior and posterior spondylolisthesis, and hypertrophy of the ligamentum flavum, can cause LFS. Because there is no standardized diagnostic imaging technique for LFS, it is 

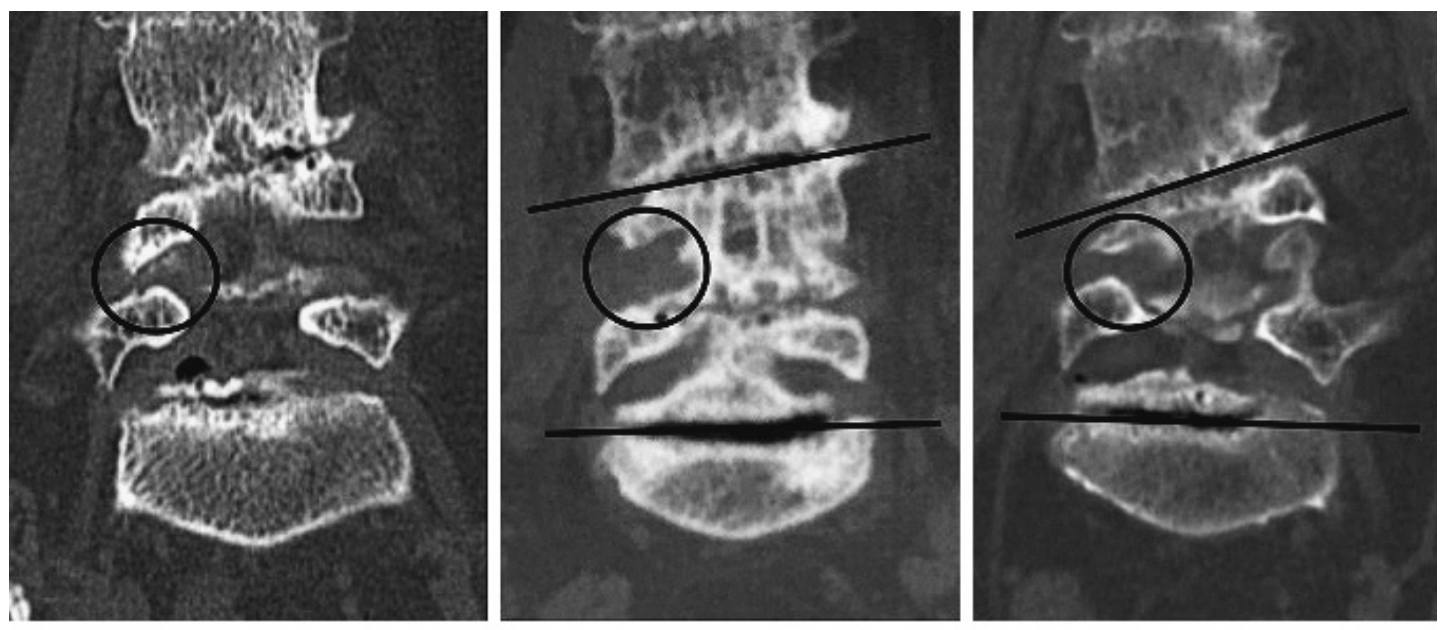

\section{A}
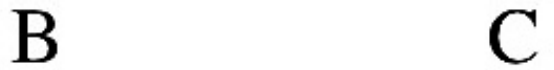

Figure 4. Coronal images of reconstructed three-dimensional computed tomography in patient 3 .

A. Before surgery.

B. Six months after surgery. Clearance between the L4 and L5 pedicles was enlarged by removing the caudal aspect of the L4 pedicle (circle).

C. Thirty-four months after surgery. Clearance between the L4 and L5 pedicles decreased because progressed degenerative scoliosis (circle).
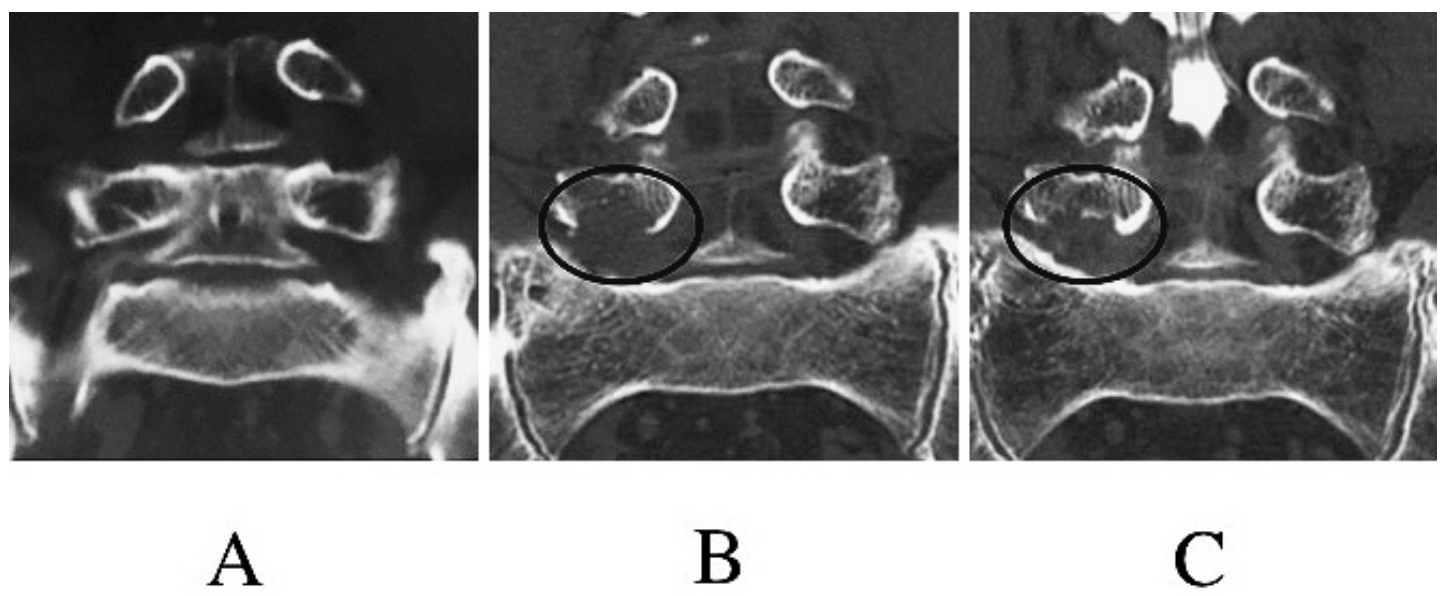

Figure 5. Coronal images of reconstructed three-dimensional computed tomography in patient 4.

A. Before surgery.

B. Six months after surgery. Clearance under the L5 pedicle was enlarged by removing the caudal aspect of the L5 pedicle (circle).

C. Thirty months after surgery. Clearance under the L5 pedicle decreased because of bone regrowth of the L5 pedicle (circle).

usually difficult to identify the nerve entrapment site. Consequently, the entire length of the nerve (nerve root, dorsal root ganglion, and spinal nerve), from the inside of the spinal canal to the outside of the intervertebral foramen, must be decompressed in most cases. Therefore, a combination of total facetectomy and spinal fusion surgery has generally been used. Although the posterior elements can be preserved by combining medial facetectomy and lateral fenestration, the nerves running under the preserved pars interarticularis cannot be decompressed with this method ${ }^{10)}$. In addition, be- cause of the deep location of the intraforaminal lesions, surgery is technically challenging and more invasive.

The major advantage of the microendoscopic technique is that the surgeon can reach deep into the body, with relatively free angles, less invasively. It is unnecessary to stick to the paraspinal posterior approach reported by Wiltse et al. ${ }^{11)}$, and the tubular retractor can be inserted from an extreme lateral position at a strongly tilted angle. Moreover, an oblique viewing endoscope makes the visual field wider. This technique facilitates decompression of the entire nerve 
Table 2. Literature Reports on Fusion Surgery for Lumbar Foraminal Stenosis.

\begin{tabular}{lcccc}
\hline \multicolumn{1}{c}{ Author } & Number of Cases & $\begin{array}{c}\text { Average Follow-up } \\
\text { Period (Months) }\end{array}$ & $\begin{array}{c}\text { Recovery Rate of } \\
\text { the JOA Score (\%) }\end{array}$ & Re-operation (\%) \\
\hline Fujibayashi et al. ${ }^{12)}$ & 16 & 23.1 & 89.1 & 6.3 \\
Xing et al. $^{14)}$ & 36 & N.A. & 31.1 & N.A. \\
Watanabe et al. $^{13)}$ & 31 & 31 & 67.1 & N.A. \\
Current study & 16 & 66.3 & 63.9 & 12.5 \\
\hline
\end{tabular}

N.A.=not available, JOA=Japanese Orthopaedic Association

length, from the spinal canal to the extraforaminal zone, without damaging posterior elements such as the facet joints and pars interarticularis. Also, using the tubular retractor minimizes muscle invasion.

Clinical results of the microendoscopic technique used in this study were favorable and similar to those of TLIF and posterior lumbar interbody fusion (PLIF), which are conven-

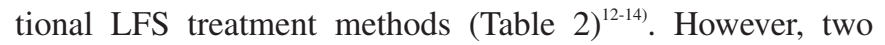
patients experienced deterioration of clinical outcomes, and revision surgery for LFS recurrence was required in two other cases.

DLS, which is common among the elderly population, is a major cause of $\mathrm{LFS}^{15)}$. Actually, of the 16 patients who were successfully followed up more than two years in this study, 5 (31.3\%) had DLS with Cobb angles of more than 10 degrees. Of these five patients, two showed increases in Cobb angle; one underwent TLIF for recurrence of LFS at the operated level, and the other suffered from low back pain with deterioration of JOA scores. The remaining three patients with DLS, however, did not show increases in Cobb angle; two maintained favorable clinical results at final follow-up, and one had recurrence of foraminal stenosis because of bone regrowth. No de-novo DLS occurred during follow-up.

DLS progression caused by degenerative change, such as lateral disc space wedging, may lead to recurrence of foraminal up-down stenosis directly. In other words, predicting curve progression is the key to selecting the current microendoscopic technique or other fusion surgeries. However, there are few reports about risk factors for curve progression after decompression surgery in the literature. With regard to LSS with DLS, Hosogane et $\mathrm{al}^{16)}$. reported that, although curve progression after decompression surgery could not be predicted from the preoperative factors, spur formation on the concave side of the curve, which might stabilize the spine, may be a candidate factor in reducing curve progression risk. Though the current technique with microendoscopy seems to maintain the coronal balance in most cases, without or with mild DLS, further studies and additional data on curve progression after decompression surgery are needed not only for LSS, but also for LFS.

Theoretically, compared to decompression alone, spinal fusion requires more aggressive intervention, requires longer operative times, and necessitates spinal implant insertion. All of these may increase the risk of complications. Therefore, it is reasonable to choose decompression alone when the pa- tient's primary symptom is neurogenic claudication, especially for elderly patients with comorbid conditions. Development of adjacent segment disease is another major issue encountered after spinal fusion. The current microendoscopic procedure seems to offer the potential for avoiding unnecessary fusion surgery in LFS cases. We recommend the current minimally invasive microendoscopic method for patients who have LFS with neurogenic claudication as a primary symptom without or with mild DLS.

There are several limitations to this study; the most significant is the lack of a control group in which LFS patients were treated with other surgical methods. The small number of patients is another shortcoming of our work. In addition, a detailed study about the frequency and degree of bone regrowth of the pedicle would optimize the degree of pedicle removal and avoid foraminal stenosis recurrence.

Conflicts of Interest: The authors declare that there are no relevant conflicts of interest.

Author Contributions: Yoshimoto wrote and prepared the manuscript, and all of the authors participated in the study design. All authors have read, reviewed, and approved the article.

\section{References}

1. Foley KT, Smith MM. Microendoscopic discectomy. Tech Neurosurg. 1997;3:301-7.

2. Yoshimoto M, Miyakawa T, Takebayashi T, et al. Microendoscopy-assisted muscle-preserving interlaminar decompression for lumbar spinal stenosis: clinical results of consecutive 105 cases with more than 3-year follow-up. Spine. 2014;39(5):E318-25.

3. Yuguchi T, Nishio M, Akiyama C, et al. Posterior microendoscopic surgical approach for the degenerative cervical spine. Neurol Res. 2003;25(1):17-21.

4. Foley KT, Smith MM, Rampersaud YR. Microendoscopic approach to far-lateral lumbar disc herniation. Neurosurg Focus. 1999;7(5):E5.

5. Matsumoto M, Chiba K, Ishii K, et al. Microendoscopic partial resection of the sacral ala to relieve extraforaminal entrapment of the L-5 spinal nerve at the lumbosacral tunnel. J Neurosurg Spine. 2006;4(4):342-6.

6. Yoshimoto M, Takebayashi T, Kawaguchi S, et al. Minimally invasive technique for decompression of lumbar foraminal stenosis using a spinal microendoscope: technical note. Minim Invasive Neurosurg. 2011;54(3):142-6.

7. Ahn Y, Oh HK, Kim H, et al. Percutaneous endoscopic lumbar foraminotomy: an advanced surgical technique and clinical out- 
comes. Neurosurgery. 2014;75(2):124-33.

8. Enyo Y, Yamada H, Kim JH, et al. Microendoscopic lateral decompression for lumbar foraminal stenosis: a biomechanical study. J Spinal Disord Tech. 2014;27(5):257-62.

9. Youn MS, Shin JK, Goh TS, et al. Clinical and radiological outcomes of endoscopic partial facetectomy for degenerative lumbar foraminal stenosis. Acta Neurochir (Wien). 2017;159(6):1129-35.

10. Kunogi J, Hasue M. Diagnosis and operative treatment of intraforaminal and extraforaminal nerve root compression. Spine. 1991; 16(11):1312-20.

11. Wiltse LL, Spencer CW. New uses and refinements of the paraspinal approach to the lumbar spine. Spine. 1988;13(6):696-706.

12. Fujibayashi S, Neo M, Takemoto $M$, et al. Paraspinal-approach transforaminal lumbar interbody fusion for the treatment of lumbar foraminal stenosis. J Neurosurg Spine. 2010;13(4):500-8.

13. Watanabe K, Yamazaki A, Morita O, et al. Clinical outcomes of posterior lumbar interbody fusion for lumbar foraminal stenosis: preoperative diagnosis and surgical strategy. J Spinal Disord Tech, 2011;24(3):137-41.

14. Xing YG, Tian W, Li Q, et al. Transforaminal lumbar interbody fusion by minimally invasive approach in the treatment of simple foraminal stenosis. Zhonghua Yi Xue Za Zhi. 2009;89(33):2342-5. Chinese.

15. Kaneko Y, Matsumoto M, Takaishi H, et al. Morphometric analysis of the lumbar intervertebral foramen in patients with degenerative lumbar scoliosis by multidetector-row computed tomography. Eur Spine J. 2012;21(12):2594-602.

16. Hosogane N, Watanabe $\mathrm{K}$, Kono $\mathrm{H}$, et al. Curve progression after decompression surgery in patients with mild degenerative scoliosis. J Neurosurg Spine. 2013;18(4):321-3.

Spine Surgery and Related Research is an Open Access journal distributed under the Creative Commons Attribution-NonCommercial-NoDerivatives 4.0 International License. To view the details of this license, please visit (https://creativeco mmons.org/licenses/by-nc-nd/4.0/). 